\title{
Feedback stabilizability of infinite dimensional neutral systems
}

\author{
S. Hadd, H. Nounou and M. Nounou
}

\begin{abstract}
A new functional analytic approach to the concept of feedback stabilizability of infinite dimensional linear neutral systems is presented. We first reformulate this systems as an infinite dimensional open-loop systems with appropriate semigroups and unbounded control operators. We introduce conditions for which such semigroups are eventually compact. In addition, when the image of the control operator is finite dimensional, we give necessary and sufficient conditions for the feedback stabilizability of neutral system. Our approach is based on the concept of regular linear systems in the Salamon-Weiss sens.
\end{abstract}

Index Terms- Neutral systems, feedback stabilizability, infinite dimensional systems, compact semigroup, Hilbert space

\section{INTRODUCTION}

In this paper, we study the concept of feedback stabilizability of partial differential equations of neutral type in infinite dimensional spaces. Before going into details we first discuss the concept of stabilizability of infinite dimensional distributed linear systems. Let $A$ be the generator of a strongly continuous semigroup $T:=(T(t))_{t \geq 0}$ on a Hilbert space $X$. This semigroup (which is the solution of the Cauchy problem $\dot{x}(t)=A x(t))$ is exponentially stable if and only if the growth bound of $T$ satisfies $\omega_{0}(A)<0$. In finite dimensional case, this is equivalent to the fact that all eigenvalues of $A$ are located in the set $\mathbb{C}_{-}:=\{\lambda \in$ $\mathbb{C}: \operatorname{Re} \lambda<0\}$. In the infinite dimensional case and if the semigroup is eventually norm continuous (so that compact semigroup) $T$ is exponentially stable if and only if the spectral growth bound of $A$ which is $s(A):=\sup \{\operatorname{Re} \lambda$ : $\lambda \in \sigma(A), \sigma(A)$ is the spectrum of $A\}<0$. This shows that regularity of the semigroup $T$ has an effect of the stability of Cauchy problems. On the other hand, the eventual compactness of the semigroup $T$ gives more information on the spectrum $\sigma(A)$ since in this case $\sigma(A)$ is countable and the set $\Lambda_{\alpha}:=\{\lambda \in \sigma(A): \operatorname{Re} \lambda \geq \alpha\}$ is finite for all $\alpha \in \mathbb{R}$. If, in addition, we assume that $\Lambda_{0}$ is not empty (which means that $T$ is unstable) then in practice it is important to shift the unstable set $\Lambda_{0}$ to $\mathbb{C}_{-}$, hence the concept of stabilizability. More generally, assume now that $T$

This work was supported by the research fund of Texas A\&M University at Qatar.

S. Hadd and H. Nounou are with the Electrical \& Computer Engineering Program, Texas A\&M University at Qatar, Doha, Qatar.

M. Nounou is with the Chemical Engineering Program, Texas A\&M University at Qatar, Doha, Qatar. is unstable and let $B: U \rightarrow X$ be a linear bounded operator ( $U$ is another Hilbert space). The stabilization problem of the open-loop system

$$
\dot{x}(t)=A x(t)+B u(t), \quad t \geq 0,
$$

consists in determining a (feedback) linear bounded operator $\Pi: X \rightarrow U$ such that the operator $A+B \Pi$ generates an exponentially stable semigroup on $X$, see [7, Chap.5]. This concept of stabilization can also be extended to unbounded control operator, that is, linear bounded operators $B$ from $U$ to $\left(\mathcal{D}\left(A^{*}\right)\right)^{\prime}$, the dual of the domain of the adjoint operator $A^{*}$, see [40, Def.2], [30].

In this paper, we consider the neutral system

$$
\left\{\begin{array}{l}
\frac{d}{d t}\left[D x_{t}\right]=A D x_{t}+L x_{t}+B u(t), \quad t \geq 0, \\
\lim _{t \rightarrow 0} D x_{t}=z \\
x(t)=\varphi(t), \quad-r \leq t \leq 0
\end{array}\right.
$$

where $A$ is the generator of a strongly continuous semigroup $T$ on a Hilbert space $X, x:[-r, \infty) \rightarrow X, r>0$, and its history function $x_{t}:[-r, 0] \rightarrow X$ is defined by $x_{t}(s)=$ $x(t+s)$ for $t \geq 0$ and $s \in[-r, 0]$, the operators $D, L$ : $W^{1,2}([-r, 0], X) \rightarrow X$ are defined by

$$
D \varphi=\varphi(0)-\int_{-r}^{0} d \mu(s) \varphi(s) \quad L \varphi=\int_{-r}^{0} d \eta(s) \varphi(s)
$$

for $\varphi \in W^{1,2}([-r, 0], X)$. The control function $u:[0, \infty) \rightarrow$ $U$ ( $U$ is a Hilbert space) is locally 2-integrable, and the control operator $B: U \rightarrow X$ are linear bounded.

The stabilization of (1) in the case when $\mu(s)=0$ for $s \in$ $[-r, 0]$, i.e. the standard delay system has been extensively studied in the literature, see e.g. [26], [22], [23], [25] in the case of $X=\mathbb{R}^{n}$ and [8], [18], [21], [24] in the case of infinite dimensional spaces.

In the case of $X=\mathbb{R}^{n}$ with generator $A \equiv 0$ and particular delay operators, see e.g. [20], [27], [29], [31]. The technique used in the so-called finite pole shifting [26]. However, the stabilizability of the neutral partial differential system (1) seems to have not yet been investigated if the space $X$ is infinite dimensional and for general delays operators (the delays are not necessary commensurable). In the present paper, we shall introduce a new approach for the stabilizability problem of (1). First, start with the stabilizability of the difference equation associated with the 
system (1). This equation can be reformulated as an openloop system with strictly unbounded control operator, see [19]. By using regular linear systems in the Salamon-Weiss sense and the definition of feedback stabilizability of openloop systems with unbounded control operators [40], we introduce a rank condition for the feedback stabilizability of the difference equation. Second, we reformulate the system (1) as an open-loop system with a linear and bounded control operator. We shall use this new system to introduce necessary and sufficient conditions for stabilizability of the neutral system (1). The necessary condition does not require any regularity on the semigroup of the transformed system. However, the sufficient conditions requires that this semigroup is eventually compact and the control operator $B$ has finite dimension.

The organization of the paper is as follows. For the reader's convenience, in Section II we briefly recall the relevant background of regular linear system from [32], [33],[39]. In Section III we present the reformulation of the neutral system (1) as a distributed linear system. In Section IV we study feedback stabilizability of the difference equation associated with (1). Section $\mathrm{V}$ is devoted to the stabilizability of the neutral system (1). In the last section we summarize the results of the paper.

\section{An overview of Salamon-Weiss systems}

For the reader's convenience, we briefly recall the concept of infinite dimensional well-posed and regular linear systems in the Salamon-Weiss sense. See [32], [33], [38], [39] for more details.

Throughout this section, $X, U$ and $Y$ are Hilbert spaces, $A: \mathcal{D}(A) \subset X \rightarrow X$ be the generator of a strongly continuous semigroup $\left(e^{t A}\right)_{t \geq 0}$ on $X$ and $1 \leq p<\infty$. We let $X_{1}^{A}$ the domain $\mathcal{D}(A)$ endowed with the graph norm and by $X_{-1}^{A}$ the completion of $X$ with respect to the norm $\|x\|_{-1}:=\left\|(\mu-A)^{-1} x\right\|$ for $x \in X$ and $\mu$ in the resolvent set $\rho(A)$. We have the following continuous injections

$$
X_{1}^{A} \hookrightarrow X \hookrightarrow X_{-1}^{A} .
$$

We note that $\left(e^{t A}\right)_{t \geq 0}$ can be extended to strongly continuous semigroup on $X_{-1}^{A}$ whose generator $A_{-1}: X \rightarrow X_{-1}^{A}$ is an extension of $A$. Define the set

$$
\begin{aligned}
& \mathfrak{B}^{p}(A, U, X)=\left\{\mathbb{B} \in \mathcal{L}\left(U, X_{-1}^{A}\right):\right. \\
& \int_{0}^{t} e^{(t-s) A_{-1}} \mathbb{B} u(s) d s \in X, \\
&\left.\quad t>0, u \in L^{p}([0, \infty), U)\right\} .
\end{aligned}
$$

The elements of this set are called admissible control oper- ators for $A$. Define the set

$$
\begin{aligned}
\mathcal{O}_{Y}^{p}(A)= & \left\{C \in \mathcal{L}\left(X_{1}^{A}, Y\right):\right. \\
& \int_{0}^{\alpha}\left\|C e^{t A} x\right\|^{p} \leq \gamma^{p}\|x\|^{p} \quad \forall x \in \mathcal{D}(A), \\
& \text { for constants } ; \alpha>0, \gamma:=\gamma(\alpha)>0\} .
\end{aligned}
$$

The elements of this set are called admissible observation operator for $A$.

Consider the input-output linear system $(A, B, C)$ described by

$$
\begin{aligned}
& \dot{z}(t)=A z(t)+\mathbb{B} u(t), \quad z(0)=z^{0}, \quad t>0, \\
& y(t)=C z(t), \quad t \geq 0,
\end{aligned}
$$

where $A: \mathcal{D}(A) \rightarrow X$ is a generator on $X, \mathbb{B} \in$ $\mathfrak{B}^{p}(A, U, X)$ and $C \in \mathcal{O}_{Y}^{p}(A)$. The functions $z:[0, \infty) \rightarrow$ $X, u:[0, \infty) \rightarrow U$ and $y:[0, \infty) \rightarrow Y$ are, respectively, the state trajectory, the control function and observation function of the system (4).

Define

$$
\Phi_{t} u=\int_{0}^{t} e^{(t-s) A_{-1}} \mathbb{B} u(\sigma) d \sigma
$$

for $t \geq 0$ and $u \in L^{p}([0, \infty), U)$. The state trajectory of the system (4) is given by

$$
z(t)=e^{t A} z^{0}+\Phi_{t} u, \quad t \geq 0 .
$$

As $C \in \mathcal{O}_{Y}^{p}(A)$, then the following operator

$$
\Psi_{\infty}: X_{1}^{A} \rightarrow L_{l o c}^{p}(\mathbb{R}+, Y), \quad x \mapsto C e^{\cdot A} x
$$

can be extended to a linear bounded operator from $X$ to $L_{\text {loc }}^{p}(\mathbb{R}+, Y)$, called the extended output map of the system

(4). The Yosida extension of $C$ for $A$ is the following operator

$$
\begin{aligned}
C_{\Lambda} x & :=\lim _{\mu \rightarrow+\infty} C \mu(\mu-A)^{-1} x \\
\mathcal{D}\left(C_{\Lambda}\right) & :=\{x \in X: \text { the above limit exists in } Y\} .
\end{aligned}
$$

Then, $\mathcal{D}(A) \subset \mathcal{D}\left(C_{\Lambda}\right)$ and that $C_{\Lambda} x=C x$ for any $x \in$ $\mathcal{D}(A)$. Further more it is shown in [35, Theorem 4.5] that

$$
e^{t A} x \in \mathcal{D}\left(C_{\Lambda}\right) \text { and }\left(\Psi_{\infty} x\right)(t)=C_{\Lambda} e^{t A} x
$$

for all $z \in X$ and almost every $t \geq 0$. Now, define

$$
\left(\Psi_{t} x\right)(\tau)=C_{\Lambda} e^{\tau A} x
$$

for $x \in X$ and $0 \leq \tau \leq t$. Hence, $\left(\Psi_{t}\right)_{t>0}$ is a family of linear bounded operators from $X$ to $L_{l o c}^{p}([0, \infty), Y)$.

Definition 2.1: We say that the system (4) is well-posed (on $X, U, Y$ ) if there exists a family $\left(\mathbb{F}_{t}\right)_{t>0}$ of linear bounded operators from $L_{l o c}^{p}([0, \infty), U)$ to $L_{l o c}^{p}([0, \infty), Y)$ such that

$$
\begin{array}{r}
{\left[\mathbb{F}_{t+s} u\right](\tau)=\left[\mathbb{F}_{t}(u(\cdot+s) \mid[0, t])\right](\tau-s)} \\
+\left[\Psi_{t} \Phi_{s}(u \mid[0, s])\right](\tau-s)
\end{array}
$$


for $\tau \in[s, s+t], t, s \geq 0$, and $u \in L^{p}([0, s+t], U)$. In this case we also say that the quadruple $\Sigma:=\left(e^{t A}, \Phi_{t}, \Psi_{t}, \mathbb{F}_{t}\right)$ is well-posed on $X, U, Y$. The operators $\mathbb{F}_{t}, t \geq 0$, are called input-output operators of $\Sigma$.

Let $\mathbf{P}_{\tau}$ be the operator of truncation to $[0, \tau]$, that is $\left(\mathbf{P}_{\tau} f\right)(t)=f(t)$ for $t \in[0, \tau]$ and zero otherwise. The operators $\mathbb{F}_{t}$ are compatible in the sense that $\mathbf{P}_{\tau} \mathbb{F}_{t}=\mathbb{F}_{\tau}$ for $t>\tau$. This property provides a unique operator $\mathbb{F}_{\infty}$ : $L_{\text {loc }}^{p}\left(\mathbb{R}_{+}, U\right) \rightarrow L_{\text {loc }}^{p}\left(\mathbb{R}_{+}, Y\right)$, called the extended inputoutput map, which satisfies $\mathbb{F}_{\tau}=\mathbf{P}_{\tau} \mathbb{F}_{\infty}=\mathbf{P}_{\tau} \mathbb{F}_{\infty} \mathbf{P}_{\tau}$ for $\tau \geq 0$.

Definition 2.2: Let $\Sigma$ be a well-posed system on $X, U, Y$ and $\mathbb{F}_{\infty}$ its extended input-output map. We say that $\Sigma$ is regular (with zero feedthrough) if the limit

$$
\lim _{t \rightarrow 0} \frac{1}{t} \int_{0}^{t}\left(\mathbb{F}_{\infty} u_{0}\right)(\tau) d \tau=0
$$

exists in $Y$ for the constant input $u_{0}(t)=z, z \in U, t \geq 0$.

The following definitions will be used throughout this paper.

Definition 2.3: We say that the triple $(A, \mathbb{B}, C)$ generates a regular system $\Sigma$ if there exists a bounded operator $\mathbb{F}_{\infty}: L_{\text {loc }}^{p}\left(\mathbb{R}_{+}, U\right) \rightarrow L_{l o c}^{p}\left(\mathbb{R}_{+}, Y\right)$ such that $\Sigma:=$ $\left(e^{t A}, \Phi_{t}, \Psi_{t}, \mathbb{F}_{t}\right)$ is regular on $Z, U, Y$.

Definition 2.4: Let $\Sigma$ be a regular system with inputoutput operators $\mathbb{F}_{t}$. An operator $\Pi \in \mathcal{L}(Y, U)$ is called an admissible feedback for $\Sigma$ if $I-\mathbb{F}_{t} \Pi$ has uniformly bounded inverse.

The following theorem is due to Weiss in Hilbert spaces [39] and to Staffans in general Banach spaces [33, Chap.7].

Theorem 2.5: Assume that $(A, \mathbb{B}, C)$ generates a regular system $\Sigma$ with admissible feedback operator $\Pi$. Then the operator defined by

$$
\begin{aligned}
A^{\Pi} & =A_{-1}+\mathbb{B} \Pi C_{\Lambda} \\
\mathcal{D}\left(A^{\Pi}\right) & :=\left\{x \in \mathcal{D}\left(C_{\Lambda}\right):\left(A_{-1}+\mathbb{B} \Pi C_{\Lambda}\right) x \in X\right\}
\end{aligned}
$$

generates a strongly continuous semigroup on $X$.

Now we give the definition of feedback stabilizability of open-loop systems with unbounded control operators.

Definition 2.6: Assume that $\mathbb{B} \in \mathfrak{B}^{p}(A, U, X)$. We say that the system

$$
\dot{x}(t)=A x(t)+\mathbb{B} u(t), \quad t \geq 0,
$$

is feedback stabilizable if there exists an operator $C$ : $\mathcal{D}(A) \rightarrow U$ such that

(i) The triple $(A, \mathbb{B}, C)$ generates a regular linear system $\Sigma$ and the identity operator $I: U \rightarrow U$ is an admissible feedback operator for $\Sigma$.

(ii) The operator

$$
\begin{aligned}
A^{I} & =A_{-1}+\mathbb{B} C_{\Lambda} \\
\mathcal{D}\left(A^{I}\right) & :=\left\{x \in \mathcal{D}\left(C_{\Lambda}\right):\left(A_{-1}+\mathbb{B} C_{\Lambda}\right) x \in X\right\}
\end{aligned}
$$

generates an exponentially stable semigroup on $X$.

III. Reformulation of the neutral system

In this section we recall the reformulation of the system (1) as an infinite dimensional distributed linear system on a product state space. To that purpose, introduce the new state space

$$
\mathcal{X}=X \times L^{2}([-r, 0], X) .
$$

Define the operator

$$
\begin{aligned}
& \mathcal{A}=\left(\begin{array}{ll}
A & L \\
0 & \frac{d}{d s}
\end{array}\right) \\
& \mathcal{D}(\mathcal{A})=\left\{\left(\begin{array}{c}
z \\
\varphi
\end{array}\right) \in \mathcal{D}(A) \times W^{1,2}([-r, 0], X):\right. \\
&\left.\varphi(0)=z+\int_{-r}^{0} d \mu(s) \varphi(s)\right\} .
\end{aligned}
$$

This operator generates a strongly continuous semigroup $\mathcal{T}:=(\mathcal{T}(t))_{t \geq 0}$ on $\mathcal{X}$, see [16]. On the other hand, the following operator

$$
\begin{aligned}
Q f & =f^{\prime}, \\
\mathcal{D}(Q) & =\left\{f \in W^{1,2}([-r, 0], X): f(0)=\right\}
\end{aligned}
$$

generates a strongly continuous semigroup $S:=(S(t))_{t \geq 0}$ on $L^{2}([-r, 0], X)$. Let $Q_{-1}$ be the extension of $Q$ in the extrapolation sense. Now define the operator

$$
\mathcal{B} u=\left(\begin{array}{c}
B u \\
0
\end{array}\right), \quad u \in U
$$

The neutral system (1) can be reformulated as the following well-posed open-loop system

$$
\left\{\begin{array}{l}
\dot{w}(t)=\mathcal{A} w(t)+\mathcal{B} u(t), \quad t \geq 0 \\
w(0)=\left(\begin{array}{c}
z \\
\varphi
\end{array}\right)
\end{array}\right.
$$

see [6].

Remark 3.1: Define the operator

$$
\mathcal{A}_{0}=\left(\begin{array}{cc}
A & L \\
0 & Q
\end{array}\right), \quad \mathcal{D}\left(\mathcal{A}_{0}\right)=\mathcal{D}(\mathcal{A}) \times \mathcal{D}(Q) .
$$

This operator is a generator of a strongly continuous semigroup on $\mathcal{X}$. Denote by $\mathcal{A}_{0,-1}$ its extension in the extrapolation sense. On the other hand define the operator

$$
\mathcal{B}_{0}=\left(\begin{array}{c}
0 \\
\left(\lambda-Q_{-1}\right) e_{\lambda}
\end{array}\right), \quad \mathcal{C}_{0}=(I K): \mathcal{D}\left(\mathcal{A}_{0}\right) \rightarrow \mathcal{X},
$$

where

$$
K \varphi=\int_{-r}^{0} d \mu(s) \varphi(s), \quad \varphi \in W^{1,2}([-r, 0], X)
$$

and $e_{\lambda}: X \rightarrow L^{2}([-r, 0], X)$ is defined by $\left(e_{\lambda} z\right)(s)=e^{\lambda s} z$ for $\lambda \in \mathbb{C}, z \in X$ and $s \in[-r, 0]$. 
We denote by $\mathcal{C}_{0, \Lambda}$ the Yosida extension of $\mathcal{C}_{0}$ for $\mathcal{A}_{0}$. It is shown in [14] and [16] that the triple $\left(\mathcal{A}_{0}, \mathcal{B}_{0}, \mathcal{C}_{0}\right)$ is a regular triple and the identity $I: X \rightarrow X$ is a feedback operator of its associated regular linear system $\Sigma$. Moreover, the operator $\mathcal{A}$ coincides with the generator of the closedloop system associated with $\Sigma$ and the feedback operator $I$. This means that

$$
\begin{aligned}
\mathcal{A} & =\mathcal{A}_{0,-1}+\mathcal{B}_{0} \mathcal{C}_{0, \Lambda}, \\
\mathcal{D}(\mathcal{A}) & =\left\{\xi \in \mathcal{D}\left(\mathcal{C}_{0, \Lambda}\right): \mathcal{A}_{0,-1} \xi+\mathcal{B}_{0} \mathcal{C}_{0, \Lambda} \xi \in \mathcal{X}\right\} .
\end{aligned}
$$

The decomposition of the generator $\mathcal{A}$ as shown in Remark 3.1 has been used in [17] to prove the following result.

Theorem 3.2: If $T(t)$ is compact for $t>0$ then $\mathcal{T}(t)$ is compact for $t>2 r$.

\section{Feedback stabilizability of difference equations}

Here we characterize the feedback stabilizability of the following difference equation associated with the neutral system (1) (in the case $A \equiv 0$ and $L \equiv 0$ )

$$
x(t)=\int_{-r}^{0} d \mu(s) x(t+s)+B u(t), \quad t \geq 0 .
$$

This is associated with the operator

$$
\begin{aligned}
Q_{\mu} f & =f^{\prime} \\
\mathcal{D}\left(Q_{\mu}\right) & =\left\{\varphi \in W^{1,2}([-r, 0], X):\right. \\
\varphi(0) & \left.=\int_{-r}^{0} d \mu(s) \varphi(s)\right\} .
\end{aligned}
$$

This operator generates a strongly continuous semigroup $S_{\mu}:=\left(S_{\mu}(t)\right)_{t \geq 0}$ in $L^{2}([-r, 0], X)$ and its resolvent set is characterized by

$$
\rho\left(Q_{\mu}\right)=\left\{\lambda \in \mathbb{C}: 1 \in \rho\left(\int_{-r}^{0} d \mu(s) e^{\lambda s}\right)\right\} .
$$

As in [19], one can see that the equation (16) can be reformulated as the well-posed open-loop system

$$
\dot{v}(t)=Q_{\mu} v(t)+\left(-Q_{-1}\right) e_{0} B u(t), \quad t \geq 0 .
$$

Now we state the main result of this section.

Theorem 4.1: The difference equation (16) is feedback stabilizable if and only if there exists $\delta>0$ such that

$$
\operatorname{Ran}\left[\Delta_{0}(\lambda) \mid B\right]=X \text { for any } \operatorname{Re} \lambda \geq-\delta
$$

where

$$
\Delta_{0}(\lambda) z=z-\int_{-r}^{0} d \mu(s) e^{\lambda s} z, \quad z \in X .
$$

Proof: First, assume that the difference equation (16) is feedback stabilizable. By using (19) and Definition 2.6, this means that there exists an operator $N: W^{1,2}([-r, 0], X) \rightarrow$ $U$ such that the following operator

$$
\begin{aligned}
Q_{\mu, N} f & =f^{\prime} \\
\mathcal{D}\left(Q_{\mu, N}\right) & =\left\{\varphi \in W^{1,2}([-r, 0], X):\right. \\
\varphi(0) & \left.=\int_{-r}^{0} d \mu(s) \varphi(s)+B N \varphi\right\}
\end{aligned}
$$

generates an exponentially stable semigroup $S_{\mu, N}$ on $L^{2}([-r, 0], X)$. Hence $s\left(Q_{\mu, N}\right) \leq \omega_{0}\left(Q_{\mu, N}\right)<0$, by [9, p.281]. Now due to (18) this is equivalent to the existence of $\delta>0$ such that $\int_{-r}^{0} d \mu(s) e^{\lambda s}+B N$ is invertible for any $\operatorname{Re} \lambda \geq-\delta$. In particular, for any $x \in X$ there exists $\vartheta \in X$ such that $\Delta_{0}(\lambda) \vartheta+B\left(-N e_{\lambda} \vartheta\right)=x$. This proves that the stabilizability of the difference equation (16) implies that $\operatorname{Ran}\left[\Delta_{0}(\lambda) \mid B\right]=X$ for $\operatorname{Re} \lambda \geq-\delta$.

\section{Feedback stabilizability of neutral systems}

Here, we present a necessary condition and a sufficient condition for the feedback stabilizability of the neutral system (1). this is equivalent to prove feedback stabilizability of the open-loop system (13). To that purpose, we need some information on the spectrum $\sigma(\mathcal{A})$. It is shown in [16] that for $\lambda \in \rho\left(Q_{\mu}\right)$ we have

$$
\sigma(\mathcal{A})=\sigma\left(A+L e_{\lambda}\left(I-\int_{-r}^{0} d \mu(s) e^{\lambda s}\right)^{-1}\right) .
$$

Define the operator

$$
\begin{aligned}
\Delta(\lambda) & :=(\lambda-A) \Delta_{0}(\lambda)-L e_{\lambda}, \\
\mathcal{D}(\Delta(\lambda)) & :=\left\{z \in X: \Delta_{0}(\lambda) z \in \mathcal{D}(A)\right\}
\end{aligned}
$$

for $\lambda \in \mathbb{C}$, where $\Delta_{0}(\lambda)$ is defined by (21). Observe that for $\lambda \in \rho\left(Q_{\mu}\right)$ we have $\lambda \in \sigma(\mathcal{A})$ if and only if $\Delta(\lambda)$ is not bijective.

Proposition 5.1: If the neutral system (1) is feedback stabilizable then there exists $\delta>0$ such that

$$
\operatorname{Ran}[\Delta(\lambda) \mid B]=X \text { and } \operatorname{Ran}\left[\Delta_{0}(\lambda) \mid B\right]=X
$$

for any $\operatorname{Re} \lambda>-\delta$.

Proof: As we have seen instead of the neutral system (1) one can consider the open-loop system $(\mathcal{A}, \mathcal{B})$ described by (13), which we assume that it is feedback stabilizable. According to [5], there exists $\delta>0$ such that

$$
\operatorname{Ran}[\lambda-\mathcal{A} \mid \mathcal{B}]=\mathcal{X} \text { for } \operatorname{Re} \lambda>-\delta
$$

Let us now show how the condition (24) together with some extra conditions on $A$ and $B$ are also sufficient for the feedback stabilizability of the neutral system (1). We assume that $A$ generates an immediately compact semigroup $T$ (i.e. $T(t)$ is compact for $t>0$ ). Theorem 3.2 shows that 
$\mathcal{A}$ generates an eventually compact semigroup $\mathcal{T}$ (i.e. there exists $t_{0}>0$ such that $\mathcal{T}(t)$ is compact for $\left.t \geq t_{0}\right)$. Hence, the spectrum $\sigma(\mathcal{A})$ is countable and the following unstable set

$$
\Omega=\{\lambda \in \sigma(\mathcal{A}): \operatorname{Re} \lambda \geq 0\}
$$

is finite, see [9, Cor.3.2, Chap.V, page 330].

Theorem 5.2: The neutral system (1) is feedback stabilizable if the following conditions are met:

(i) A generates an immediately compact semigroup,

(ii) the dimension of the image $\operatorname{ImB}$ is finite, and

(iii) the condition (24) is satisfied for all $\lambda \in \Omega$.

Proof: Let $\left(\begin{array}{l}x \\ f\end{array}\right) \in \mathcal{X}$ and put

$$
\Omega=\left\{\lambda_{1}, \cdots, \lambda_{k}\right\} .
$$

First, we assume that $\Omega \subset \rho\left(Q_{\mu}\right)$. We shall use a result of [5], which gives condition for stabilizability of systems having compact semigroups. For $i=1, \cdots, k$, there exists $\varphi \in \rho\left(Q_{\mu}\right)$ such that $\lambda_{i} \varphi-\varphi^{\prime}=f$. From (24), there exists $z_{0} \in X$ and $u_{0} \in U$ such that

$$
\begin{aligned}
& \left(\lambda_{i}-A\right) z-L e_{\lambda_{i}} z_{0}+B u_{0} \\
& \quad=x+L R\left(\lambda_{i}, Q\right) f+L e_{\lambda_{i}} \varphi(0),
\end{aligned}
$$

where $z=\Delta_{0}\left(\lambda_{i}\right) z_{0} \in \mathcal{D}(A)$. We now introduce

$$
\psi=e_{\lambda_{i}} z_{0}+\varphi
$$

then we have

$$
\left(\begin{array}{l}
z \\
\psi
\end{array}\right) \in \mathcal{D}(\mathcal{A}) \text { and } \quad \lambda_{i} \psi-\psi^{\prime}=f .
$$

From (25)-(26) and the fact that $\varphi=e_{\lambda_{i}} \varphi(0)+R\left(\lambda_{i}, Q\right) f$ we have

$$
\begin{aligned}
\left(\lambda_{i}-A\right) & z-L \psi+B u_{0} \\
= & x+L e_{\lambda_{i}} K e_{\lambda_{i}} z_{0} .
\end{aligned}
$$

On the other hand, there exists $y_{0} \in X$ and $u_{1} \in U$ such that

$$
\left(\lambda_{i}-A\right) y-L e_{\lambda_{i}} y_{0}+B u_{1}=L e_{\lambda_{i}} K e_{\lambda_{i}} z_{0},
$$

where $y=\Delta_{0}\left(\lambda_{i}\right) y_{0} \in \mathcal{D}(A)$. If we put $g:=\psi-e_{\lambda_{i}} y_{0}$, $w:=z-y$ and $u=u_{0}-u_{1}$ then by (27) and (28) we have

$$
\left(\lambda_{i}-A\right) w-L g+B u=x \text { and } \lambda_{i} g-g^{\prime}=f .
$$

Observe that $\left(\begin{array}{c}w \\ g\end{array}\right) \in \mathcal{D}(\mathcal{A})$ and then $\left(\lambda_{i}-\mathcal{A}\right)\left(\begin{array}{c}w \\ g\end{array}\right)+\mathcal{B} u=$ $\left(\begin{array}{l}x \\ f\end{array}\right)$. Since, by Theorem 3.2, $\mathcal{A}$ generates an eventually compact semigroup on $\mathcal{X}$, then by [5] the open-loop system $(\mathcal{A}, \mathcal{B})$ is feedback stabilizable.

Second, let us assume that there exists a set $\Theta \subsetneq \Omega$ such that $\Theta \cap \rho\left(Q_{\mu}\right)=\{\emptyset\}$. As in the proof of Theorem 4.1, the second condition in (24) implies that there exists an operator $N: W^{1,2}([-r, 0], X) \rightarrow X$ such that the semigroup $S_{\mu, N}$ solution of the difference equation $x(t)=(K+B N) x_{t}$ is exponentially stable. If we denote by $Q_{\mu, N}$ the generator of $S_{\mu, N}$ then we have $\Theta \subset \rho\left(Q_{\mu, N}\right)$. Now we consider the feedback law $u(t)=\frac{d}{d t} N x_{t}+v(t)$, where $v(t)$ is a new input. It suffices then to stabilize the system

$$
\left\{\begin{array}{l}
\frac{d}{d t}\left[D x_{t}-B N x_{t}\right]=A D x_{t}+L x_{t}+B u(t), \quad t \geq 0, \\
\lim _{t \rightarrow 0} D x_{t}=z \\
x(t)=\varphi(t), \quad-r \leq t \leq 0 .
\end{array}\right.
$$

We then proceed as in the first step to shift the unstable set $\Theta$ to $\mathbb{C}_{-}$. This ends the proof.

Example 5.3: Let $A$ be the generator of an immediately compact semigroup on a Hilbert space $X,\left(M_{i}\right)_{1 \leq i \leq m}$ be a family of bounded linear operators on $X$, and $C: X \rightarrow X$ be a linear and bounded operator. Consider the neutral system

$$
\left\{\begin{array}{c}
\dot{x}(t)-\sum_{i=1}^{i=m} M_{i} \dot{x}(t-r)= \\
A\left[x(t)-\sum_{i=1}^{i=m} M_{i} x(t-r)\right] \\
+C x(t-r)+B u(t), \quad t \geq 0, \\
\lim _{t \rightarrow 0}\left[x(t)-\sum_{i=1}^{i=m} M_{i} x(t-r)\right]=z, \\
x(t)=\varphi(t), \quad \text { a.e. } \quad-r \leq t \leq 0,
\end{array}\right.
$$

with $0<r_{1}<r_{2}<\cdots<r_{m} \leq r$. After defining the difference operator $D \varphi=\varphi(0)-\sum_{i=1}^{i=m} M_{i} \varphi\left(r_{i}\right)$ and the operators $K, L: W^{1,2}([-r, 0], X) \rightarrow X$ by $K \varphi=$ $\sum_{i=1}^{i=m} M_{i} \varphi\left(r_{i}\right)$ and $L \varphi=C \varphi(-r)$, the system (30) corresponds to the neutral system (1). Now by Proposition 5.1 and Theorem 5.2, the neutral system (30) is feedback stabilizable if and only if

$$
\operatorname{Ran}\left[I-\sum_{i=1}^{i=m} e^{\lambda r_{i}} M_{i} \mid B\right]=X
$$

and there exist $x \in X$ and $u \in U$ such that $x-$ $\sum_{i=1}^{i=m} e^{\lambda r_{i}} M_{i} x \in \mathcal{D}(A)$ and

$$
\left(\lambda_{A}\right)\left(x-\sum_{i=1}^{i=m} e^{\lambda r_{i}} M_{i} x\right)-e^{-\lambda r} C x+B u=z
$$

for all $z \in X$ and $\lambda \in \Omega$.

\section{Conclusion}

In this paper, we have presented a new functional analytic approach to stabilize a class of neutral systems. We first presented a necessary and sufficient condition for the feedback stabilizability of difference systems (see Theorem 4.1). Such systems are reformulated as distributed linear systems with strictly unbounded control operators. We have then used Salamon-Weiss systems to prove Theorem 4.1. On the other hand, to stabilize the neutral system (1) we have reformulated this system as a linear system $(\mathcal{A}, \mathcal{B})$ with a linear bounded control operator. In order to use a well-known result on the 
stabilization of the evolution systems we have introduced conditions for which the operator $\mathcal{A}$ generates an eventually compact semigroup.

\section{References}

[1] A. Bensoussan, G. Da Prato, M. C. Delfour and S. K. Mitter, Representation and Control of Infinite-Dimensional Systems. Birkhäuser, Boston, Basel, Berlin, 2007.

[2] A. Bátkai and S. Piazzera, Semigroups for Delay Equations. Research Notes in Mathematics, 10. A K Peters, Ltd., Wellesley, MA, 2005.

[3] Boulite, S., Idrissi, A. and Maniar, L., Robustness of controllability under unbounded perturbations, J. Math. Anal. Appl. 304 (2005), 409421.

[4] R. Bellman and K.L. Cooke, Differential-Difference Equations. Academic Press, New York, 1963.

[5] K.P.M. Bhat and W.M. Wonham, Stabilizability and detectability for evolution systems on Banach spaces, in Proc. IEEE Conference on Decision Control, Institute of Electrical and Electronics Engineers, New York, 1976, pp. 1240-1234.

[6] H. Bounit, S. Hadd, Regular linear systems governed by neutral FDEs, Journal Mathematical Analysis Applications. 320(2), 836-858, 2006.

[7] R.F. Curtain and H. Zwart, Introduction to Infinite-Dimensional Linear Systems. TMA 21, Springer-Verlag, New York, 1995.

[8] G. Da Prato and A. Lunardi, Stabilization of integrodifferential parabolic equations, J. Integral Equations Appli. 2:281-304, 1990.

[9] K.-J. Engel and R. Nagel, One-Parameter Semigroups for Linear Evolution Equations. Springer-Verlag, 2000.

[10] S. Hadd, Unbounded perturbations of $\mathcal{C}_{0}$-semigroups in Banach spaces and applications, Semigroup Forum. vol. 70, no. 3, pp. 451465, 2005

[11] S. Hadd, Exact controllability of infinite dimensional systems persists under small perturbations, Journal of Evolution Equations, 5 (2005), $545-555$.

[12] S. Hadd and A. Idrissi, Regular linear systems governed by systems with state, input and output delays, IMA Journal Mathematical Control and Information. vol. 22, no. 4, pp. 423-439, 2005.

[13] S. Hadd and A. Idrissi, On the admissibility of observation for perturbed $C_{0}$-semigroups on Banach spaces, Systems Control Letters. vol. 55, no. 1, 1-7, 2006.

[14] S. Hadd, A. Idrissi and A. Rhandi, The regular linear systems associated to the shift semigroups and application to control delay systems, Mathematical Control Signals Systems. vol. 18, no. 3, pp. 272-291, 2006.

[15] H. Leiva, Unbounded perturbation of the controllability for evolution equations, J. Math. Anal. Appl. 280 (2003), 1-8.

[16] S. Hadd and A. Rhandi, Feedback theory for neutral equations in infinite dimensional spaces. Note Matematica, 1:43-68, 2008.

[17] S. Hadd, H. Nounou and M. Nounou, Eventual norm continuity of the neural semigroup on Banach spaces, J. Math. Anal. Appl. Accepted.

[18] S. Hadd and Q-C. Zhong, On feedback stabilizability of linear systems with state and input delays in Banach spaces. IEEE trans. Automat. Control, 54:438-451, 2009.

[19] S. Hadd and Q-C. Zhong, Perturbation theory of boundary value problems and approximate controllability of perturbed boundary control problems, the 47th IEEE CDC, 2008, 1465-1470.
[20] J.K. Hale and S.M. Verduyn Lunel, Introduction to Functional Differential Equations. plus 0.5em Applied Mathematical Sciences, vol. 99, Springer-Verlag, New York, 1993.

[21] J.-M. Jeong, Retarded functional differential equations with $L^{1}$-valued controller," Funkcial Ekvac., vol. 36, pp. 71-93, 1993.

[22] A. Manitius and R. Triggiani, "Sufficient conditions for function space controllability and feedback stabilizability of linear retared systems," IEEE Trans. Automat. Control, AC-23, pp. 659-664, 1978.

[23] A. Manitius and R.W. Olbrot, "Finite spectrum assignment problem for systems with delays," IEEE Transaction Automatic Control. AC24, pp. 541-553, 1979 .

[24] S-I Nakagiri and M. Yamamoto, Feedback stabilization of linear retarded systems in Banach spaces. Journal Mathematical Analysis Applications. vol. 262, no. 1, pp. 160-178, 2001.

[25] A.W. Olbrot, Stabilizability, detectability, and spectrum assignment for linear autonomous systems with general time delays, IEEE Transaction Automatic Control. vol. AC-23, no. 5, pp. 887-890, 1978.

[26] L. Pandolfi, Feedback stabilization of functional differential equations. Boll. Un. Mat. Ita., vol. 11, no. 3, pp. 626-635, 1975.

[27] L. Pandolfi, Stabilization of neutral functional equations. Journal Optimization Theory Application. vol. 20, no. 2, pp. 191-204, 1996.

[28] L. Pandolfi, Dynamic stabilization of systems with input delays. Automatica Journal IFAC. vol. 27, no. 6, 1047-1050, 1991

[29] D.A. O'Connor and T.J. Tran, On stabilization by state feedback for neutral differential-difference equations. IEEE Transaction Automatic Control. vol. AC-28, no. 5, pp. 615-618, 1983.

[30] R. Rebarber, Necessary conditions for exponential stabilizability of distributed parameter systems with infinite dimensional unbounded feedback. Systems Control Letters, vol. 14, no. 3, pp. 241-248, 1990.

[31] D. Salamon, Control and Observation of Neutral Systems. Pitam, Boston, 1984

[32] D. Salamon, Infinite-dimentional linear system with unbounded control and observation: a functional analytic approach. Transaction American Mathematical Society. vol. 300, no. 2, pp. 383-431, 1987.

[33] O. J. Staffans, Well-Posed Linear Systems. Cambridge Univ. Press, 2005.

[34] M. Tucsnak and G. Weiss, Observation and Control for Operator Semigroups, Birkhäuser, 2009.

[35] G. Weiss, Admissible observation operators for linear semigoups Israel Journal Mathematics. vol. 65, no. 1, pp. 17-43, 1989.

[36] G. Weiss, Admissibility of unbounded control operators. SIAM Journal Control Optimization. vol. 27, no. 3, pp. 527-545, 1989.

[37] G. Weiss, Two conjectures on the admissibility of control operators, in: W. Desch, F. Kappel (Eds.), Estimation and Control of Distributed Parameter Systems, Birkhäuser, Basel, 1991, pp. 367-378.

[38] G. Weiss, Transfer functions of regular linear systems. Part I: Characterization of regularity. Transaction American Mathematical Society. vol. 342, no. 2, pp. 827-854, 1994.

[39] G. Weiss, Regular linear systems with feedback, Mathematical Control Signals Systems. vol. 7, no. 1, pp. 23-57, 1994.

[40] G. Weiss and R. Rebarber, Optimizability and estimatability for infinite-dimensional linear systems. SIAM Journal Control Optimization. vol. 39, no. 4 , pp. 1204-1232, 2000. 